\title{
A TRANSMISSION PROBLEM IN THE SCATTERING OF ELECTROMAGNETIC WAVES BY A PENETRABLE OBJECT*
}

\author{
RODOLFO H. TORRES ${ }^{\dagger}$
}

\begin{abstract}
Layer-potential techniques are used to study a transmission problem arising in the scattering of electromagnetic waves by a penetrable object. The method proposed does not involve the use of the calculus of pseudodifferential operators and hence it can be applied in domains with very little regularity. The solutions are represented as a combination of a curl and a double curl of a single layer-potential operator. The work relies on the important harmonic-analysis tools developed in recent years to study boundary-value problems in domains with minimal regularity assumptions.
\end{abstract}

Key words. Maxwell equations, reduced wave equation, layer-potential methods, transmission problems, scattering theory, nonsmooth domains

AMS subject classifications. 35J05, 35Q60, 45P05, 58G20, 31A25

1. Introduction. A classical problem arising in electromagnetism is that of determining the field scattered by a penetrable object from the knowledge of the tangential component on the surface of the object of an incoming field. See, e.g., [12] and [15]. The mathematical formulation of this problem leads to a transmission problem for the Maxwell equations on a bounded domain (see $\S 2$ below for the precise statement). The problem has been studied using several approaches based on layerpotentials techniques. In particular, we want to mention works by Wilde [20] and Costabel and Stephan [5]. Reference to related works can be found therein.

For time-harmonic electromagnetic waves, the solution of Maxwell equations are divergence-free solutions of the vector Helmholtz equation. In [20], very general transmission problems for the vector Helmholtz equation are considered. The solutions of the problems are obtained as a combination of several single- and double-layer potentials after solving, in appropriate Hölder spaces, a $4 \times 4$ system of of integral equations of the second kind on the boundary of the domain. This classical method requires the domain to be at least of class $C^{2}$ and, as a consequence, the solutions have continuous partial derivatives up to the boundary of the domain. On the other hand, in [5], the so-called direct method is used. This is a general method applicable to strongly elliptic boundary-value problems and relies on the coercivity on certain Sobolev spaces (the energy spaces) of a bilinear form related to the boundary data. In [5], the electromagnetic problem is transformed into a particular transmission problem for the vector Helmholtz equation which is solved, again, by inverting a matrix of operators on the boundary of the domain. In this work, the calculus of pseudodifferential operators is used and hence the domain is assumed to be $C^{\infty}$. In addition, the boundary values of the solutions are prescribed in the distributional sense and not pointwise. The purpose of this paper is to develop an alternative approach to study the electromagnetic transmission problem in domains which are less regular than the one considered in the works just mentioned, allowing less regular boundary data, but still obtaining solutions whose boundary values are prescribed pointwise (nontangentially).

As is well known, the study of boundary-value problems using layer-potential techniques in domains which are $C^{1}$ or Lipschitz is very delicate. One of the main reasons for this is that some of the resulting integral operators on the boundary of the

\footnotetext{
* Received by the editors May 6, 1994; accepted for publication February 16, 1995. This research was supported in part by NSF grant DMS-9303363.

$\dagger$ Department of Mathematics, University of Michigan, Ann Arbor, MI 48109. Current address: Department of Mathematics, University of Kansas, Lawrence, KS 66045-2142.
} 
domain have to be interpreted as principal-value singular integrals. In particular, to consider $L^{p}$ data and solutions with boundary values obtained pointwise, deep results from harmonic analysis are necessary. Dirichlet and conormal derivative problems for several equations and system of equations in nonsmooth domains have already been studied using harmonic-analysis techniques. A few examples are [9], [11], [17], [18], [7]. Using similar techniques, transmission problems have been considered in [8] and [16]. This last paper deals with the case of the scalar Helmholtz equation in Lipschitz domains. See also [19] and [6], where an approach to transmission problems related to $[5]$ is used.

The study of the potential operators associated with Maxwell equations in $C^{1}$ and Lipschitz domains has been recently carried out in [13] and [14]. In particular, the so-called Maxwell, electric, and magnetic boundary-value problems for a perfect conducting object were solved with optimal estimates in the case of $C^{1}$ domains. This work depends heavily on the results in [2] and [3] about the Cauchy integral operator on Lipschitz curves as well as the developments in [9]. For the previously known results about these problems in the case of smoother domains, we refer to [4].

In this paper, we will combine the results of [13] with some of the ideas in [16] to study the electromagnetic transmission problem in domains which are only $C^{1}$ or Lipschitz. Unlike the approaches in [20] and [5], we propose as a solution for the electromagnetic transmission problem a combination of the curl and the curlcurl of the single-layer potential. After taking traces, this ansatz leads to a $2 \times 2$ system of integral operators on the boundary of the domain. The trace operator associated with the double curl of the single-layer potential is hypersingular (even on smooth domains). Nevertheless, in the case of the electromagnetic transmission problem, this operator appears in a regularized way. This allows us to consider it on an appropriate space of functions: the space $L_{T}^{2 \text {,Div }}$ consisting of tangential vector fields with surface divergence in $L^{2}$. It was shown in [14] that $L_{T}^{2, \text { Div }}$ is the right space of boundary data to work with in domains with little regularity: As in [16], the solution of the system of integrals operators on the boundary relies on the knowledge of the spectrum of a singular integral operator. In our present situation, the singular-integral operator is the one obtained as the tangential component of the trace of the curl of the singlelayer potential.

The paper is organized as follows. In $\S 2$, we recall some basic facts about nonsmooth domains and state the transmission problem with boundary data in $L_{T}^{2, \text { Div }}$. In $\S 3$, we show for appropriate values of the electromagnetic characteristics of the object and surrounding media the uniqueness of solution to the problem in the case of Lipschitz domains. In $\S 4$, we collect several results from [13] about the layer-potential operators associated with Maxwell equations and include some new results regarding the double curl of the single-layer potential. In $\S 5$, we show some existence results.

2. The electromagnetic transmission problem. The notation that we use is standard for the subject. In particular, we will follow very closely that of [13] which is our main reference. For the purposes of this paper, a Lipschitz, respectively, $C^{1}$, domain will always be an open, simply connected domain $D$ of $\mathrm{R}^{3}$, whose boundary, $\partial D$, is given locally by the graph of a Lipschitz, respectively, $C^{1}$, function. Let $N$ be the exterior unit normal to $\partial D$ and let $d \sigma$ denote surface measure on the boundary. The spaces $L^{2}(\partial D)$ of functions or vector fields and the space $L_{T}^{2}(\partial D)$ of tangential vector fields are defined with respect to $d \sigma$. The space $L^{2,1}(\partial D)$ is, as usual, the space of $L^{2}$ functions with tangential derivatives also in $L^{2}$. A vector field $A \in L_{T}^{2}(\partial D)$ is 
said to have a surface divergence if there exists a function $b \in L^{2}(\partial D)$ such that

$$
\int_{\partial D}\left\langle\nabla_{T} \psi, A\right\rangle d \sigma=-\int_{\partial D} \psi b d \sigma
$$

for all functions $\psi$ which are Lipschitz in a neighborhood of $\partial D$. Here $\nabla_{T}$ denotes the tangential gradient and $\langle\cdot, \cdot\rangle$ denotes the inner product in $\mathrm{R}^{3}$. The function $b$ is denoted by $\operatorname{Div} A$ and the space of all such vector fields (see, e.g., [14]) is denoted by $L_{T}^{2, \text { Div }}(\partial D)$. The space is equipped with the norm

$$
\|A\|_{L_{T}^{2, \mathrm{Div}}(\partial D)}=\|A\|_{L^{2}(\partial D)}+\|\operatorname{Div} A\|_{L^{2}(\partial D)} .
$$

At every point $Q$ in the boundary of the domain, we consider an open, rightcircular, doubly truncated cone $\Gamma(Q)$, with vertex at $Q$ and two convex components, $\Gamma_{i}(Q)$ in $D$ and $\Gamma_{e}(Q)$ in $\mathrm{R}^{3} \backslash \bar{D}$, so that the resulting family of cones is a regular family in the sense of [17]. For a function $u$ defined in $D$, the nontangential maximal function of $u$ is defined by

$$
u^{*}(P)=\sup _{X \in \Gamma_{i}(P)}|u(X)|
$$

The boundary values of functions defined inside $D$ are assumed to be taken in nontangential fashion and almost everywhere with respect to $d \sigma$. That is, $\left.u\right|_{\partial D}$ is to be interpreted as

$$
u(P)=\lim _{\substack{X \rightarrow P \\ X \in \Gamma_{i}(P)}} u(X)
$$

whenever such a limit exists for almost every point in $\partial D$. Similar definitions apply for derivatives of a function and for each component of a vector-valued function. For example, if $\times$ denotes the exterior product in $\mathrm{R}^{3}$ and $A$ is a vector field defined inside $D$, then $N \times\left.\operatorname{curl} A\right|_{\partial D}$ is given by

$$
N \times \operatorname{curl} A(P)=\lim _{\substack{X \rightarrow \Gamma_{i} \\ X \in \Gamma_{i}(P)}} N(P) \times \operatorname{curl} A(X) .
$$

For functions defined in the exterior of $D$, the nontangential maximal function and the boundary values are defined in the same way but using $\Gamma_{e}(P)$.

We can now state the electromagnetic transmission problem that we want to study. We follow the classical description in [15]. Let $D$ represent an object made of an homogeneous material, and assume that the object is immersed in an homogeneous medium represented by the exterior of $D$. In all space, we consider a time-harmonic electromagnetic wave with frequency $\omega$, described by the electric and magnetic vector fields $E$ and $H$. These fields satisfy the Maxwell equations

$$
\begin{gathered}
\operatorname{curl} E=i \omega \mu_{i} H \\
\operatorname{curl} H=-i \omega \epsilon_{i} E \text { in } D,
\end{gathered}
$$

and

$$
\operatorname{curl} E=i \omega \mu_{e} H \text {, }
$$




$$
\operatorname{curl} H=-i \omega \epsilon_{e} E \quad \text { in } \mathrm{R}^{3} \backslash \bar{D} \text {. }
$$

The electromagnetic parameters of the object and the surrounding medium in the above equations are, respectively,

$$
\begin{aligned}
\epsilon_{i} & =\epsilon_{0 i}+\frac{i \sigma_{i}}{\omega}, & \mu_{i} & =\mu_{0 i}+\frac{i \hat{\sigma}_{i}}{\omega}, \\
\epsilon_{e} & =\epsilon_{0 e}+\frac{i \sigma_{e}}{\omega}, & \mu_{e} & =\mu_{0 e}+\frac{i \hat{\sigma}_{e}}{\omega},
\end{aligned}
$$

where $\epsilon_{0 i}$ and $\epsilon_{0 e}$ are the dielectric constants, $\mu_{0 i}$ and $\mu_{0 e}$ are the permeability, $\sigma_{i}$ and $\sigma_{e}$ are the electric conductivity, and $\hat{\sigma}_{i}$ and $\hat{\sigma}_{e}$ are the magnetic conductivity of each medium. The usual restrictions on the values of these parameters are

$$
\begin{gathered}
0 \leq \arg \omega<\pi \\
\epsilon_{0 i}, \epsilon_{0 e}>0 \text { and } \mu_{0 i}, \mu_{0 e}>0 \\
\sigma_{i}, \sigma_{e} \geq 0 \text { and } \hat{\sigma}_{i}, \hat{\sigma}_{e} \geq 0
\end{gathered}
$$

(see [15]). We will assume $\epsilon_{i} \neq \epsilon_{e}$ and $\mu_{i} \neq \mu_{e}$. The wave numbers in the interior and exterior of the obstacle are defined by

$$
k_{i}^{2}=\omega^{2} \epsilon_{i} \mu_{i} \quad \text { and } \quad k_{e}^{2}=\omega^{2} \epsilon_{e} \mu_{e}
$$

where we assume

$$
0 \leq \arg k_{i}, \quad \arg k_{e}<\pi .
$$

In the exterior of $D$, the vector fields are decomposed as the sum of a known incoming field and an unknown scattered field,

$$
\begin{aligned}
& E=E_{\text {in }}+E_{\mathrm{sc}}, \\
& H=H_{\text {in }}+H_{\text {sc }} .
\end{aligned}
$$

Both the incoming and scattered fields satisfy Maxwell's equations in the exterior of $D$. We also assume that the scattered fields satisfy the radiation conditions

$$
\omega \mu_{e} \frac{X}{|X|} \times H_{\mathrm{sc}}+k_{e} E_{\mathrm{sc}}=o\left(|X|^{-1}\right) \text { and } E_{\mathrm{sc}}=O\left(|X|^{-1}\right)
$$

as $|X| \rightarrow \infty$. The tangential components of the total vector fields must extend continuously across the boundary, so on $\partial D$ we must have

$$
\begin{aligned}
& N \times E-N \times E_{\mathrm{sc}}=N \times E_{\mathrm{in}}, \\
& N \times H-N \times H_{\mathrm{sc}}=N \times H_{\mathrm{in}},
\end{aligned}
$$

where the values of $N \times E$ and $N \times H$ are taken from inside $D$. It follows that, in order to obtain the total electric and magnetic fields from the knowledge of the 
incoming fields, we can consider a transmission boundary-value problem with the tangential components of the incoming fields as datum. Because of the results in [13] and [14], we will assume that these tangential components are in $L_{T}^{2 \text {,Div }}(\partial D)$, and we will require the solutions to have nontangential maximal functions bounded on $L^{2}(\partial D)$. In fact, it was shown in [14] that if $E$ and $H$ solve Maxwell equations in a $C^{1}$ or Lipschitz domain and $E$ has pointwise (nontangentially) boundary values in $L^{2}(\partial D)$ with bounded nontangential maximal function, then the companion field $H$ also have pointwise boundary values if and only if the tangential component of $E$ is in $L_{T}^{2, \text { Div }}(\partial D)$. Since the roles of $E$ and $H$ can be interchanged, we have to require the same kind of boundary data for the tangential component of $H$. Thus we are lead to consider the following problem. Given two tangential vector fields $A$ and $B$ in $L_{T}^{2 \text {,Div }}(\partial D)$, find two vector fields in $D, E_{i}$ and $H_{i}$, and two vector fields in $\mathrm{R}^{3} \backslash \bar{D}$, $E_{e}$ and $H_{e}$, satisfying the radiation condition (5) and such that

$$
(T) \begin{cases}\operatorname{curl} E_{i}=i \omega \mu_{i} H_{i} & \text { in } D, \\ \operatorname{curl} H_{i}=-i \omega \epsilon_{i} E_{i} & \text { in } D, \\ \left\|E_{i}^{*}\right\|_{L^{2}(\partial D)}+\left\|H_{i}^{*}\right\|_{L^{2}(\partial D)}<\infty, \\ \operatorname{curl} E_{e}=i \omega \mu_{e} H_{e} & \text { in } \mathrm{R}^{3} \backslash \bar{D} \\ \operatorname{curl} H_{e}=-i \omega \epsilon_{e} E_{e} & \text { in } \mathrm{R}^{3} \backslash \bar{D}, \\ \left\|E_{e}^{*}\right\|_{L^{2}(\partial D)}+\left\|H_{e}^{*}\right\|_{L^{2}(\partial D)}<\infty, \\ N \times E_{e}-N \times E_{i}=A & \text { on } \partial D, \\ N \times H_{e}-N \times H_{i}=B & \text { on } \partial D .\end{cases}
$$

3. Uniqueness of solution. The uniqueness of solution of problem $(T)$ is given in [12] and [15] for smooth domains and functions continuous up to the boundary. We will consider here the case of Lipschitz domains, boundary data in $L_{T}^{2 \text {,Div }}(\partial D)$, and boundary values obtained nontangentially. We will always assume that the electromagnetic parameters satisfy the constrains in (1)-(4). Additional limitations in their values will be imposed, if necessary, in the statments of the results to be proved.

Usually, the proof of uniqueness results for boundary-value problems involves integral-representation formulas and some application of the divergence theorem. The standard technique to adapt these formulas to the case of nonsmooth domains is an approximation procedure. The main tool is the following lemma from [17].

Lemma 3.1. Let $D$ be a bounded Lipschitz domain. Then it is possible to construct a sequence of $C^{\infty}$ domains $\Omega_{j} \subset D$ (or $\Omega_{j} \supset D$ ) satisfying the following properties:

(i) There is a sequence of Lipschitz diffeomorphisms $\Lambda_{j}: \partial D \rightarrow \partial \Omega_{j}$. such that the Lipschitz constants of $\Lambda_{j}$ and its inverse are uniformly bounded in $j$. Furthermore, $\Lambda_{j}(Q) \in \Gamma_{i}(Q)\left(\right.$ or $\left.\Gamma_{e}(Q)\right)$ for all $j$ and all $Q \in \partial D$ and $\sup _{Q \in \partial D}\left|Q-\Lambda_{j}(Q)\right| \leq C / j$;

(ii) There are positive functions $\rho_{j}: \partial D \rightarrow R_{+}$bounded away from zero and infinity uniformly in $j$ such that for any measurable set $F \subset \partial D, \int_{F} \rho_{j} d \sigma=\int_{\Lambda_{j}(F)} d \sigma_{j}$ and such that $\rho_{j} \rightarrow 1$ a.e. and in every $L^{p}(\partial D), 1 \leq p<\infty$;

(iii) The sequence of normal vectors to $\Omega_{j}, N_{j}\left(\Lambda_{j}(\cdot)\right)$ converges a.e. and in every $L^{p}(\partial D), 1 \leq p<\infty$, to $N$.

Let $k$ be a complex number with $\operatorname{Im} k \geq 0$ and consider the fundamental solution of the Helmholtz operator $\triangle+k^{2}$ in $\mathrm{R}^{3}$,

$$
\Phi(X)=-\frac{e^{i k|X|}}{4 \pi|X|} .
$$


We will need to use the following formulae regarding solutions of the vector Helmholtz equation.

LEMMA 3.2. Let $D$ be a Lipschitz domain and let $E$ be a smooth vector field in $D$ or $R^{3} \backslash \bar{D}$. Assume that $E$, curl $E$, and $\operatorname{div} E$ have nontangentially boundary values on $\partial D$ from the inside or the outside accordingly to where $E$ is defined. Assume also that

$$
\left\|E^{*}\right\|_{L^{2}(\partial D)}+\left\|(\operatorname{div} E)^{*}\right\|_{L^{2}(\partial D)}+\left\|(\operatorname{curl} E)^{*}\right\|_{L^{2}(\partial D)}<\infty .
$$

The following formulas hold:

(i) The tangential vector field $N \times E$ has a surface divergence in $L^{2}(\partial D)$ and

$$
\operatorname{Div}(N \times E)=-\langle N, \operatorname{curl} E\rangle .
$$

(ii) If $E$ is a solution of the vector Helmholtz equation $\triangle E+k^{2} E=0$ in $D$, then for all $X \in D$,

$$
\begin{aligned}
E(X)= & -\int_{\partial D} \operatorname{curl}_{X}(\Phi(X-Q) N(Q) \times E(Q)) d \sigma \\
& +\int_{\partial D} \nabla_{X} \Phi(X-Q)\langle N(Q), E(Q)\rangle d \sigma \\
& -\int_{\partial D} \Phi(X-Q)(N(Q) \times \operatorname{curl} E(Q)-\operatorname{div} E(Q) N(Q)) d \sigma
\end{aligned}
$$

and

$$
\begin{gathered}
\int_{\partial D}\langle N(Q) \times \bar{E}(Q), \operatorname{curl} E(Q)\rangle+\operatorname{div} E(Q)\langle N(Q), \bar{E}(Q)\rangle d \sigma \\
=\int_{D}|\operatorname{curl} E(X)|^{2}+|\operatorname{div} E(X)|^{2}-k^{2}|E(X)|^{2} d X .
\end{gathered}
$$

(iii) If $E$ is a solution of the vector Helmholtz equation $\triangle E+k^{2} E=0$ in $R^{3} \backslash \bar{D}$ that satisfies at infinity the radiation condition

$$
\operatorname{curl} E \times \frac{X}{|X|}+\operatorname{div} E \frac{X}{|X|}-i k E=\mathrm{o}\left(|X|^{-1}\right), \quad E=O\left(|X|^{-1}\right),
$$

then for all $X \in R^{3} \backslash \bar{D}$,

$$
\begin{aligned}
E(X)= & \int_{\partial D} \operatorname{curl}_{X}\{\Phi(X-Q) N(Q) \times E(Q)\} d \sigma \\
& -\int_{\partial D} \nabla_{X} \Phi(X-Q)\langle N(Q), E(Q)\rangle d \sigma \\
& +\int_{\partial D} \Phi(X-Q)(N(Q) \times \operatorname{curl} E(Q)-\operatorname{div} E(Q) N(Q)) d \sigma
\end{aligned}
$$

and

$$
\lim _{r \rightarrow \infty}\left(-\int_{|X|=r}|k|^{2}|E(X)|^{2}+|\operatorname{curl} E(X) \times N(X)+\operatorname{div} E(X) N(X)|^{2} d s_{r}\right.
$$




$$
\begin{gathered}
\left.-2 \operatorname{Im}(k) \int_{D_{r}}|\operatorname{curl} E(X)|^{2}+|\operatorname{div} E(X)|^{2}+|k|^{2}|E(X)|^{2} d X\right) \\
=2 \operatorname{Im}\left(k \int_{\partial D}(\langle N(Q), E(Q) \times \operatorname{curl} \bar{E}(Q)\rangle+\operatorname{div} \bar{E}(Q)\langle N(Q), E(Q)\rangle) d \sigma\right),
\end{gathered}
$$

where $d s_{r}$ is the surface measure on the ball of radius $r, B_{r}(0)$, and where $D_{r}=$ $R^{3} \backslash \bar{D} \cap B_{r}(0)$.

Proof. The above formulas are well known for smooth domains. The validity of them in the case of Lipschitz domains was justified in [14] using Lemma 3.1 and a limiting argument. We shall not repeat the details here (cf. the proof of Theorem 3.4 below).

A simple consequence of the above lemma is the following result.

LEMMA 3.3. Let $D$ be a Lipschitz domain. Let $E$ be a solution of the vector Helmholtz equation in $R^{3} \backslash \bar{D}$ satisfying (6), the radiation condition (8), and the inequality

$$
\operatorname{Im}\left(k \int_{\partial D}(\langle N(Q), E(Q) \times \operatorname{curl} \bar{E}(Q)\rangle+\operatorname{div} \bar{E}(Q)\langle N(Q), E(Q)\rangle) d \sigma\right) \geq 0 .
$$

If $\operatorname{Im} k>0$, then $E=0$ in $R^{3} \backslash \bar{D}$.

Proof. If $\operatorname{Im} k>0$, then from the last part of Lemma 3.2,

$$
\int_{D_{r}}|E(X)|^{2} d X \rightarrow 0
$$

which implies that $E=0$.

We can now prove a uniqueness results for solutions of the transmission problem $(T)$. Recall that solutions of the Maxwell equations

$$
\begin{gathered}
\operatorname{curl} E=i \omega \mu H, \\
\operatorname{curl} H=-i \omega \epsilon E
\end{gathered}
$$

are divergence-free solutions of the vector Helmholtz equation with wave number $k^{2}=\omega^{2} \epsilon \mu$. Notice also the equivalence between the radiation conditions (5) and (8).

TheOREm 3.4. Let $D$ be a Lipschitz domain. Assume that $\operatorname{Im} k_{i}>0$ and $\operatorname{Im} k_{e}>$ 0 , and let $E_{i}, H_{i}, E_{e}$, and $H_{e}$ be solutions of $(T)$ with boundary data $A=B=0$. Then $E_{i}=H_{i}=0$ in $D$ and $E_{e}=H_{e}=0$ in $R^{3} \backslash \bar{D}$.

Proof. We will use a limiting argument to adapt the proof in [15, p. 282], for the case of smooth domains to the present situation. Let $\Omega_{j}$ be a family of domains approximating $D$ from inside as in Lemma 3.1. Since solutions of Maxwell equations are analytic inside $D$ we can apply the divergence theorem on each domain $\Omega_{j}$. We obtain

$$
\begin{gathered}
\int_{\Omega_{j}}\left(i \omega \epsilon_{i}\left|E_{i}\right|^{2}-i \overline{\omega \mu_{i}}\left|H_{i}\right|^{2}\right) d X=\int_{\Omega_{j}} \operatorname{div}\left(\bar{E}_{i} \times H_{i}\right) d X \\
=\int_{\partial \Omega_{j}}\left\langle N_{j}, \bar{E}_{i} \times H_{i}\right\rangle d \sigma_{j}
\end{gathered}
$$


and using the change of coordinates $\Lambda_{j}$,

$$
\begin{gathered}
\int_{\Omega_{j}}\left(i \omega \epsilon_{i}\left|E_{i}\right|^{2}-i \overline{\omega \mu_{i}}\left|H_{i}\right|^{2}\right) d X \\
=\int_{\partial D}\left\langle N_{j}\left(\Lambda_{j}(Q)\right), \bar{E}_{i}\left(\Lambda_{j}(Q)\right) \times H_{i}\left(\Lambda_{j}(Q)\right)\right\rangle \rho_{j} d \sigma .
\end{gathered}
$$

The integrals on the left of the above equality are uniformly bounded by

$$
C \int_{\partial D}\left|E_{i}^{*}(Q)\right|\left|H_{i}^{*}(Q)\right| d \sigma
$$

Since we are assuming that $\left\|E_{i}^{*}\right\|_{L^{2}(\partial D)}+\left\|H_{i}^{*}\right\|_{L^{2}(\partial D)}<\infty$, we can use the properties of the approximating domains together with the dominated-convergence theorem to get

$$
\int_{D}\left(i \omega \epsilon_{i}\left|E_{i}\right|^{2}-i \overline{\omega \mu_{i}}\left|H_{i}\right|^{2}\right) d X=\int_{\partial D}\left\langle N, \bar{E}_{i} \times H_{i}\right\rangle d \sigma .
$$

A similar argument in the exterior of $D$ shows that

$$
\begin{gathered}
\int_{D_{r}}\left(i \omega \epsilon_{i}\left|E_{e}\right|^{2}-i \overline{\omega \mu_{i}}\left|H_{e}\right|^{2}\right) d X \\
=\int_{X=r}\left\langle\frac{X}{r}, \bar{E}_{e} \times H_{e}\right\rangle d s-\int_{\partial D}\left\langle N, \bar{E}_{e} \times H_{e}\right\rangle d \sigma .
\end{gathered}
$$

Adding the formulas for the interior and exterior, using the transmission conditions with $A=B=0$ and the radiation condition at infinity, we get

$$
\begin{gathered}
\int_{D}\left(i \omega \epsilon_{i}\left|E_{i}\right|^{2}-i \overline{\omega \mu_{i}}\left|H_{i}\right|^{2}\right) d X+\int_{D_{r}}\left(i \omega \epsilon_{i}\left|E_{e}\right|^{2}-i \overline{\omega \mu_{i}}\left|H_{e}\right|^{2}\right) d X \\
=\int_{X=r}\left\langle\frac{X}{r}, \bar{E}_{e} \times H_{e}\right\rangle d s \\
=\frac{\bar{k}_{e}}{\overline{\omega \mu_{e}}} \int_{X=r}\left|E_{e}\right|^{2} d s+o(1) .
\end{gathered}
$$

Now, by the constraints on the electromagnetic parameters,

$$
\operatorname{Re}(i \omega \epsilon) \leq 0 \text { and } \operatorname{Re}(\overline{i \omega \mu)} \geq 0,
$$

where $\epsilon$ denotes either $\epsilon_{i}$ or $\epsilon_{e}$ and $\mu$ denotes either $\mu_{i}$ or $\mu_{e}$. In addition,

$$
\operatorname{Re}\left(\frac{k_{e}}{\omega \mu_{e}}\right) \geq 0
$$

It follows that we must have

$$
\operatorname{Re}\left(\int_{D}\left(i \omega \epsilon_{i}\left|E_{i}\right|^{2}-i \overline{\omega \mu_{i}}\left|H_{i}\right|^{2}\right) d X\right)=0
$$




$$
\lim _{r \rightarrow \infty} \operatorname{Re}\left(\int_{D_{r}}\left(i \omega \epsilon_{i}\left|E_{e}\right|^{2}-i \overline{\omega \mu_{i}}\left|H_{e}\right|^{2}\right) d X\right)=0,
$$

and

$$
\lim _{r \rightarrow \infty} \operatorname{Re}\left(\frac{k_{e}}{\omega \mu_{e}}\right) \int_{X=r}\left|E_{e}\right|^{2} d s=0 .
$$

Moreover, since we are assuming $\operatorname{Im} k_{i}>0$, one of the parameters $\omega, \epsilon_{i}$, and $\mu_{i}$ is not a real number. Then either $\operatorname{Re}\left(i \omega \epsilon_{i}\right)<0$ or $\operatorname{Re}\left(-i \overline{\omega \mu_{i}}\right)<0$. From (9), one of the fields vanishes in $D$ and so both $E_{i}$ and $H_{i}$ must be identically zero in $D$. Finally, from the transmission conditions, the tangential components of $E_{e}$ and $H_{e}$ on the boundary have to be zero and, since $\operatorname{Im} k_{e}>0$, Lemma 3.3 implies that $E_{e}$ and $H_{e}$ must be identically zero in the exterior of $D$.

Remark. The conditions $\operatorname{Im} k_{i}>0$ and $\operatorname{Im} k_{e}>0$ in the above theorem are removed in [15] for the case of smooth domains by a more elaborated argument. Nevertheless, we will still need those conditions to prove existence of solutions.

We conclude this section with another uniqueness result. As we will see in the proof of existence of solutions, the transmission problem in the next theorem can be used, in a general sense, as adjoint problem for problem (T) (cf. [6]).

Theorem 3.5. Let $D$ be a Lipschitz domain in $R^{3}$. Assume that $\operatorname{Im} k_{i}>0$ and $\operatorname{Im} k_{e}>0$. Assume also that either

$$
\begin{gathered}
\operatorname{Im}\left(k_{i} \bar{k}_{e}^{2} \frac{\mu_{e}}{\mu_{i}}\right) \leq 0, \\
\operatorname{Im}\left(k_{i} \frac{\mu_{e}}{\mu_{i}}\right) \geq 0
\end{gathered}
$$

or

$$
\begin{gathered}
\frac{\epsilon_{e} \mu_{i}}{\mu_{e}}=\epsilon_{0 e}^{\prime}+\frac{i \sigma_{e}^{\prime}}{\omega} \quad \text { with } \epsilon_{0 e}^{\prime}>0 \text { and } \sigma_{e}^{\prime} \geq 0, \\
\frac{\mu_{e} \epsilon_{i}}{\epsilon_{e}}=\mu_{0 e}^{\prime}+\frac{i \hat{\sigma}_{e}^{\prime}}{\omega} \quad \text { with } \mu_{0 e}^{\prime}>0 \text { and } \hat{\sigma}_{e}^{\prime} \geq 0 .
\end{gathered}
$$

Then the homogeneous transmission problem for the vector Helmholtz equation,

$$
\left(T^{\prime}\right) \begin{cases}\triangle E_{i}+k_{e}^{2} E_{i}=0 & \text { in } D, \\ \operatorname{div} E_{i}=0 & \text { in } D, \\ \left\|E_{i}^{*}\right\|_{L^{2}(\partial D)}+\left\|\left(\operatorname{curl} E_{i}\right)^{*}\right\|_{L^{2}(\partial D)}<\infty, & \quad \text { in } R^{3} \backslash \bar{D}, \\ \triangle E_{e}+k_{i}^{2} E_{e}=0 \quad \text { in } R^{3} \backslash \bar{D}, \\ \operatorname{div} E_{e}=0 & \text { on } \partial D, \\ \left\|E_{e}^{*}\right\|_{L^{2}(\partial D)}+\left\|\left(\operatorname{curl} E_{e}\right)^{*}\right\|_{L^{2}(\partial D)}<\infty, & \text { on } \partial D, \\ N \times E_{e}-N \times E_{i}=0 & \end{cases}
$$

where $E_{e}$ satisfies the radiation condition (8) with $k=k_{i}$, has the unique solution $E_{i}=0$ in $D$ and $E_{e}=0$ in $R^{3} \backslash \bar{D}$. 
Proof. Assume that conditions (12) and (13) are satisfied. Let $E_{i}$ and $E_{e}$ be solutions of problem $\left(T^{\prime}\right)$. Using the transmission conditions and the divergence theorem (whose used can be justified again via Lemma 3.1 and the boundedness of nontangential maximal functions), we get

$$
\begin{aligned}
\int_{\partial D}\left\langle N, E_{e} \times \operatorname{curl} \bar{E}_{e}\right\rangle d \sigma & =\int_{\partial D}\left\langle N, E_{i} \times \overline{\left(\frac{k_{i}^{2} \mu_{e}}{k_{e}^{2} \mu_{i}}\right)} \operatorname{curl} \bar{E}_{i}\right\rangle d \sigma \\
& =\int_{D}\left(\overline{\left(\frac{k_{i}^{2} \mu_{e}}{k_{e}^{2} \mu_{i}}\right)}\left|\operatorname{curl} E_{i}\right|^{2}-\overline{\left(\frac{k_{i}^{2} \mu_{e}}{\mu_{i}}\right)}\left|E_{i}\right|^{2}\right) d X .
\end{aligned}
$$

Now using the constraints on the electromagnetic parameters, we see that

$$
\operatorname{Im}\left(k_{i} \int_{\partial D}\left\langle N, E_{e} \times \operatorname{curl} \bar{E}_{e}\right\rangle d \sigma\right) \geq 0 .
$$

Since $\operatorname{div} E_{e}=0$, Lemma 3.3 implies that $E_{e}=0$. Again using the transmission conditions and the representation formula in Lemma 3.2 for the interior of $D$, we also obtain that $E_{i}=0$.

Assume now that conditions (14) and (15) are satisfied. Let $E_{i}$ and $E_{e}$ again be solutions of the problem $\left(T^{\prime}\right)$. Then it follows that $E_{i}, H_{i}=1 / i \omega \mu_{i}^{\prime}$ curl $E_{i}$ and $E_{e}$, $H_{e}=1 / i \omega \mu_{e}^{\prime} \operatorname{curl} E_{e}$ are solutions of the homogeneous version of problem $(T)$ with electromagnetic parameters $\epsilon_{i}^{\prime}=\epsilon_{e}$ and $\mu_{i}^{\prime}=\mu_{e}$ in the interior and $\epsilon_{e}^{\prime}=\epsilon_{e} \mu_{i} / \mu_{e}$ and $\mu_{e}^{\prime}=\mu_{e} \epsilon_{i} / \epsilon_{e}$ in the exterior. By Theorem 3.4, $E_{i}$ and $E_{e}$ must be zero.

Remark. The conditions on the electromagnetic parameters in the above theorem look very technical because we have stated the result in great generality. If some of the parameters are real valued, these conditions become much simpler. See Theorem 5.2 below.

4. Boundary integral operators. We recall some properties about the layerpotential operators associated with the Helmholtz and Maxwell equations. The results are well known for smooth domains; see, e.g., [4]. For nonsmooth domains, we refer for proofs and details to [1] and [16] for the case of the scalar Helmholtz equation and to $[14]$ for the vector-valued case.

Let $D$ be a Lipschitz domain and let $f$ be a function in $L^{2}(\partial D)$. The single and double acoustic layer potentials are given by

$$
\mathcal{S} f(X)=\int_{\partial D} \Phi(X-Q) f(Q) d \sigma(Q), \quad X \in \mathrm{R}^{3},
$$

and

$$
\mathcal{D} f(X)=\int_{\partial D} \partial_{N_{Q}} \Phi(X-Q) f(Q) d \sigma(Q), \quad X \in \mathrm{R}^{3} \backslash \partial D .
$$

Both $\mathcal{S} f$ and $\mathcal{D} f$ solve the Helmholtz equation in $\mathrm{R}^{3} \backslash \partial D$ and, as a consequence of the results in [3], they satisfy

$$
\left\|(\mathcal{S} f)^{*}\right\|_{L^{2}(\partial D)}+\left\|(\nabla \mathcal{S} f)^{*}\right\|_{L^{2}(\partial D)}+\left\|(\mathcal{D} f)^{*}\right\|_{L^{p}(\partial D)} \leq C\|f\|_{L^{2}(\partial D)} .
$$

The trace values of $\mathcal{S} f$ are given by

$$
\lim _{\substack{X \rightarrow \Gamma_{X} \\ X \in \Gamma_{i}(P)}} \mathcal{S} f(X)=\lim _{\substack{X \rightarrow \Gamma^{X} P \\ X \in \Gamma_{e}(P)}} \mathcal{S} f(X)=S f(P), \quad P \in \partial D,
$$


where

$$
S f(P)=-\frac{1}{4 \pi} \int_{\partial D} \frac{e^{i k|Q-P|}}{|Q-P|} f(Q) d \sigma(Q), \quad P \in \partial D .
$$

The function $\mathcal{D} f$ has a jump discontinuity given by

$$
\begin{aligned}
& \lim _{\substack{X \rightarrow P \\
X \in \Gamma_{i}(P)}} \mathcal{D} f(X)=\left(\frac{1}{2} I+K\right) f(P), \quad P \in \partial D, \\
& \lim _{\substack{X \rightarrow P \\
X \in \Gamma_{e}(P)}} \mathcal{D} f(X)=\left(-\frac{1}{2} I+K\right) f(P), \quad P \in \partial D .
\end{aligned}
$$

where

$$
K f(P)=\frac{1}{4 \pi} \text { p.v. } \int_{\partial D} \frac{\langle N(Q), Q-P\rangle}{|Q-P|^{3}} e^{i k|Q-P|}(1-i k|Q-P|) f(Q) d \sigma(Q) .
$$

The normal derivative of the single-layer potential satisfies

$$
\lim _{\substack{X \rightarrow \Gamma_{i}(P) \\ X \in \Gamma_{i}}}\langle N(P), \nabla \mathcal{S} f(X)\rangle=\left(-\frac{1}{2} I+K^{*}\right) f(P)
$$

and

$$
\lim _{\substack{X \rightarrow \Gamma_{e}(P) \\ X \in \Gamma_{e}(P)}}\langle N(P), \nabla \mathcal{S} f(X)\rangle=\left(\frac{1}{2} I+K^{*}\right) f(P),
$$

where $K^{*}$ is the transpose operator of $K$. On the other hand, the tangential component of $\nabla \mathcal{S} f$ does not jump.

For the rest of the section, we will assume that the imaginary part of the wave number $k$ is positive. This condition guarantees the invertibility results in the next lemma (see [1], [16]).

LEMMA 4.1. Let $D$ be a Lipschitz domain in $R^{3}$. Then the following hold:

(i) $S: L^{2}(\partial D) \longrightarrow L^{2}(\partial D)$ is compact.

(ii) $S: L^{2}(\partial D) \longrightarrow L^{2,1}(\partial D)$ is invertible.

(iii) $\pm \frac{1}{2} I+K: L^{2}(\partial D) \longrightarrow L^{2}(\partial D)$ are invertible.

(iv) $\pm \frac{1}{2} I+K: L^{2,1}(\partial D) \longrightarrow L^{2,1}(\partial D)$ are invertible.

(v) If $\partial D$ is actually of class $C^{1}$, then the operator $K$ is compact in $L^{2}(\partial D)$.

The action of the single and double layer-potential operators on vector fields is defined componentwise. In addition, the traces of the divergence and curl of the single-layer potential of a vector field $A$ define bounded operators in $L^{2}(\partial D)$, and their values are given by

$$
\lim _{\substack{X \rightarrow P \\ X \in \Gamma_{i}(P)}} \operatorname{div} \mathcal{S} A(X)=-\frac{1}{2}\langle N, A\rangle(P)+\text { p.v. } \int_{\partial D} \operatorname{div}_{P}(\Phi(P-Q) A(Q)) d \sigma(Q),
$$

$$
\lim _{\substack{X \rightarrow P \\ X \in \Gamma_{e}(P)}} \operatorname{div} \mathcal{S} A(X)=\frac{1}{2}\langle N, A\rangle(P)+\text { p.v. } \int_{\partial D} \operatorname{div}_{P}(\Phi(P-Q) A(Q)) d \sigma(Q),
$$


and

$$
\begin{aligned}
& \lim _{\substack{X \rightarrow P \\
X \in \Gamma_{i}(P)}} \operatorname{curl} \mathcal{S} A(X)=-\frac{1}{2}(N \times A)(P)+\text { p.v. } \int_{\partial D} \operatorname{curl}_{P}(\Phi(P-Q) A(Q)) d \sigma(Q), \\
& \lim _{\substack{X \rightarrow P \\
X \in \Gamma_{e}(P)}} \operatorname{curl} \mathcal{S} A(X)=\frac{1}{2}(N \times A)(P)+\text { p.v. } \int_{\partial D} \operatorname{curl}_{P}(\Phi(P-Q) A(Q)) d \sigma(Q) .
\end{aligned}
$$

We also have

$$
\left\|(\operatorname{div} \mathcal{S} A)^{*}\right\|_{L^{2}(\partial D)}+\left\|(\operatorname{curl} \mathcal{S} A)^{*}\right\|_{L^{2}(\partial D)} \leq C\|A\|_{L^{2}(\partial D)}
$$

The function curl $\mathcal{S} A$ satisfies the vector Helmholtz equation outside $\partial D$ as well as the radiation condition (8) at infinity. In addition, the tangential component of the trace of the curl of the single-layer potential is given almost everywhere in $\partial D$ by

$$
\lim _{\substack{X \rightarrow P \\ X \in \Gamma_{i}(P)}} N(P) \times \operatorname{curl} \mathcal{S} A(X)=\left(\frac{1}{2} I+M\right) A(P)
$$

and

$$
\lim _{\substack{X \rightarrow \Gamma_{e}(P) \\ X \in \Gamma_{e}}} N(P) \times \operatorname{curl} \mathcal{S} A(X)=\left(-\frac{1}{2} I+M\right) A(P),
$$

where $M A$ is the tangential vector field defined by

$$
M A(P)=\text { p.v. } \int_{\partial D} N(P) \times \operatorname{curl}_{P}(\Phi(P-Q) A(Q)) d \sigma(Q) .
$$

We recall from [13] the following result.

LeMmA 4.2. Let $D$ be a Lipschitz domain in $R^{3}$. Then the operator $M$ maps $L_{T}^{2}(\partial D)$ into itself and $L_{T}^{2 \text {,Div }}(\partial D)$ into itself. Moreover, if $D$ is actually $C^{1}$, then $M$ is compact on both spaces.

In order to study the double curl of the single-layer potential, we need another important result obtained in [14].

Lemma 4.3. Let $D$ be Lipschitz domain. A vector field $A$ in $L_{T}^{2}(\partial D)$ has a surface divergence in $L^{2}(\partial D)$ if and only if $\left\|(\nabla(\operatorname{div} \mathcal{S} A))^{*}\right\|_{L^{2}(\partial D)}<+\infty$. In such a case, $\operatorname{div} \mathcal{S} A=\mathcal{S}(\operatorname{Div} A)$.

As a consequence of this last result, we can now prove the following.

LeMma 4.4. Let $D$ be a Lipschitz domain. Let $A$ be a vector field in $L_{T}^{2, \text { Div }}(\partial D)$. Then $\left\|(\operatorname{curl} \operatorname{curl} \mathcal{S} A)^{*}\right\|_{L^{2}(\partial D)}<+\infty$ and

$$
\lim _{X \rightarrow P} N(P) \times \operatorname{curl} \operatorname{curl} \mathcal{S} A(X)=\left(N \times\left(k^{2} \mathcal{S} A+\nabla \mathcal{S}(\operatorname{Div} A)\right)\right)(P)
$$

nontangentially, both from the inside and outside of $D$. Moreover, if we define on $\partial D$ the operator

$$
L A(P)=\left(N \times\left(k^{2} \mathcal{S} A+\nabla \mathcal{S}(\operatorname{Div} A)\right)\right)(P)
$$

then $L$ maps $L_{T}^{2, \text { Div }}(\partial D)$ into itself. 
Proof. Let $A$ be a vector field in $L_{T}^{2, \text { Div }}(\partial D)$. Using the identity

$$
\operatorname{curl} \operatorname{curl}=-\Delta+\nabla \operatorname{div}
$$

and Lemma 4.3 , we see that

$$
\operatorname{curl} \operatorname{curl} \mathcal{S} A=k^{2} \mathcal{S} A+\nabla \mathcal{S}(\operatorname{Div} A),
$$

which implies the boundedness of the nontangential maximal function and the claimed boundary values (notice that $\mathcal{S}$ and the tangential component of $\nabla \mathcal{S}$ do not have jumps). Clearly, the resulting boundary operator $L$ maps $L_{T}^{2, \text { Div }}(\partial D)$ into $L_{T}^{2}(\partial D)$. If we now apply (7) to the vector field

$$
E(X)=\operatorname{curl} \operatorname{curl} \mathcal{S} A(X),
$$

we obtain that

$$
\operatorname{Div}(L A)=\operatorname{Div}(N \times E)=-\langle N, \operatorname{curl} \operatorname{curl} \operatorname{curl} \mathcal{S} A\rangle=-\left\langle N, k^{2} \operatorname{curl} \mathcal{S} A\right\rangle,
$$

and, therefore,

$$
\begin{aligned}
\|L A\|_{L_{T}^{2, \operatorname{Div}}(\partial D)} & =\|L A\|_{L_{T}^{2}(\partial D)}+\|\operatorname{Div}(L A)\|_{L^{2}(\partial D)} \\
& \leq\left\|N \times\left(k^{2} \mathcal{S} A+\nabla \mathcal{S}(\operatorname{Div} A)\right)\right\|_{L_{T}^{2}(\partial D)}+\left\|\left\langle N, k^{2} \operatorname{curl} \mathcal{S} A\right\rangle\right\|_{L^{2}(\partial D)} \\
& \leq C\left(\|\mathcal{S} A\|_{L^{2}(\partial D)}+\|\nabla \mathcal{S}(\operatorname{Div} A)\|_{L^{2}(\partial D)}+\|\operatorname{curl} \mathcal{S} A\|_{L^{2}(\partial D)}\right) \\
& \left.\leq C\left(\|A\|_{L_{T}^{2}(\partial D)}+\| \operatorname{Div} A\right) \|_{L^{2}(\partial D)}\right)
\end{aligned}
$$

which concludes the proof.

We need to consider the potential-theoretic versions of some of the layer-potential operators already described. Let $S_{0}, M_{0}$, and $L_{0}$ be defined using the fundamental solution of the Laplace operator $\triangle$ in $\mathrm{R}^{3}$,

$$
\Phi_{0}(X)=-\frac{1}{4 \pi|X|} .
$$

The boundedness properties of the operators $S_{0}, M_{0}$, and $L_{0}$ are the same as those of $S, M$, and $L$. Moreover, we have the following.

LEMMA 4.5. Let $D$ be a Lipschitz domain in $R^{3}$. Then,

(i) $M-M_{0}: L_{T}^{2}(\partial D) \longrightarrow L_{T}^{2}(\partial D)$ is compact.

(ii) $M-M_{0}: L_{T}^{2}(\partial D) \longrightarrow L_{T}^{2, \text { Div }}(\partial D)$ is bounded.

(iii) $L-L_{0}: L_{T}^{2}(\partial D) \longrightarrow L_{T}^{2}(\partial D)$ is compact.

(iv) $L-L_{0}: L_{T}^{2}(\partial D) \longrightarrow L_{T}^{2, \text { Div }}(\partial D)$ is bounded.

Proof. A straightforward computation shows that the differences of partial derivatives

$$
\partial_{i} \Phi(P-Q)-\partial_{i} \Phi_{0}(P-Q)
$$

and

$$
\partial_{i} \partial_{j} \Phi(P-Q)-\partial_{i} \partial_{j} \Phi_{0}(P-Q)
$$

have locally integrable singularities on $\partial D$. From this easily follows that $M-M_{0}$ and $L-L_{0}$ are compact operators in $L_{T}^{2}(\partial D)$ (cf. [16]). To show that these operators map $L_{T}^{2}(\partial D)$ into $L_{T}^{2, \text { Div }}(\partial D)$, we notice that

$$
\begin{aligned}
\operatorname{Div}\left(M-M_{0}\right) A & =\operatorname{Div}\left(N \times \operatorname{curl}\left(\mathcal{S}-\mathcal{S}_{0}\right) A\right) \\
& =-\left\langle N, \operatorname{curl} \operatorname{curl}\left(\mathcal{S}-\mathcal{S}_{0}\right) A\right\rangle \\
& =-\left\langle N, k^{2} \operatorname{curl} \mathcal{S} A+\nabla \operatorname{div}\left(\left(\mathcal{S}-\mathcal{S}_{0}\right) A\right)\right\rangle
\end{aligned}
$$


which defines an operator with a kernel with a locally integrable singularity and bounded in $L^{2}(\partial D)$. Similarly,

$$
\begin{aligned}
\operatorname{Div}\left(L-L_{0}\right) A & =\operatorname{Div}\left(N \times \operatorname{curl} \operatorname{curl}\left(\mathcal{S}-\mathcal{S}_{0}\right) A\right) \\
& =-\left\langle N, \operatorname{curl} \operatorname{curl} \operatorname{curl}\left(\mathcal{S}-\mathcal{S}_{0}\right) A\right\rangle \\
& =-\left\langle N, k^{2} \operatorname{curl} \mathcal{S} A\right\rangle,
\end{aligned}
$$

again producing a bounded operator in $L^{2}(\partial D)$.

We conclude this section with a simple result about the spectrum of $M_{0}$ in $C^{1}$ domains.

LEMma 4.6. Let $D$ be a $C^{1}$ domain in $R^{3}$. Then, for any complex number $\lambda$ outside the interval $\left[-\frac{1}{2}, \frac{1}{2}\right]$, the operator $\lambda I+M_{0}$ is invertible in $L_{T}^{2}(\partial D)$ and in $L_{T}^{2, \text { Div }}(\partial D)$.

Proof. Since by the results in [14] the operator $M_{0}$ still is $L^{2}$-compact in $C^{1}$ domains, it is enough to prove that $\lambda I+M_{0}$ is injective. This is done in [4, pp. 155$157]$ in the case of $C^{2}$ domains. Given the boundedness and invertibility properties of the layer-potential operators discussed in this section, the same proof extends without modification to the case of $C^{1}$ domains. Finally, observe that since $M_{0}$ is also compact in $L_{T}^{2, \text { Div }}(\partial D)$, its spectrum in $L_{T}^{2}(\partial D)$ and in $L_{T}^{2, \text { Div }}(\partial D)$ is the same.

Remark. In the case of Lipschitz domains, the operators $M$ and $M_{0}$ may not be compact in $L_{T}^{2}(\partial D)$. The invertibility of $\lambda I+M$ or $\lambda I+M_{0}$ can no longer be handled via Fredholm theory. The usual substitute technique to prove invertibility results in this kind of situation involves the use of Rellich-type identities (see, e.g., [17] and [7]). Such techniques were used in [8] and [10] to study the spectrum in $L^{2}(\partial D)$ of the double-layer potential for the Laplacian $\lambda I+K_{0}$. The spectral properties of $K_{0}$ in $L^{2,1}(\partial D)$ were studied in [16]. The spectral properties of $M_{0}$ in $L_{T}^{2}(\partial D)$ in the case of Lipschitz domains remain unknown, but from the results in [13], it follows that if $\lambda I+M_{0}$ is in vertible in $L_{T}^{2}(\partial D)$ for some $\lambda$, then it is also invertible in $L_{T}^{2, \text { Div }}(\partial D)$. This missing information about the spectrum of $M_{0}$ in $L_{T}^{2}(\partial D)$ is the only additional result that would be necessary to extend Theorem 5.1 in the next section to the case of Lipschitz domains.

5. Existence of solutions. We now present the existence of solutions to problem $(T)$ using a particular boundary integral representation.

TheOREM 5.1. Let $D$ be a $C^{1}$ domain in $R^{3}$. Assume that the electromagnetic parameters satisfy the conditions in Theorem 3.5. Assume also that $\frac{\mu_{e}+\mu_{i}}{\mu_{e}-\mu_{i}}$ and $\frac{\epsilon_{e}+\epsilon_{i}}{\epsilon_{e}-\epsilon_{i}}$ are not real numbers in the interval $[-1,1]$. Then the transmission problem $(T)$ has a unique solution for any $A$ and $B$ in $L_{T}^{2, \text { Div }}(\partial D)$.

Proof. In view of Theorem 3.4, we only need to show existence of solution. Let $U$ and $V$ be vector fields in $L_{T}^{2, D i v}(\partial D)$ and consider the ansatz

$$
\begin{gathered}
E_{e}(X)=\mu_{e} \operatorname{curl} \mathcal{S}_{e} U(X)+\operatorname{curl} \operatorname{curl} \mathcal{S}_{e} V(X) \text { in } \mathrm{R}^{3} \backslash \bar{D} \\
E_{i}(X)=\mu_{i} \operatorname{curl} \mathcal{S}_{i} U(X)+\operatorname{curl} \operatorname{curl} \mathcal{S}_{i} V(X) \text { in } D
\end{gathered}
$$

and

$$
\begin{aligned}
& H_{e}(X)=\frac{1}{i \mu_{e} \omega} \operatorname{curl} E_{e}(X), \\
& H_{i}(X)=\frac{1}{i \mu_{e} \omega} \operatorname{curl} E_{i}(X),
\end{aligned}
$$


where $\mathcal{S}_{e}$ and $\mathcal{S}_{i}$ denote the single layer-potential operators defined using the wave numbers $k_{e}$ and $k_{i}$. By the results of the previous section, for any $U$ and $V$ in $L_{T}^{2, \text { Div }}(\partial D)$, these vector fields solve the Maxwell equations and satisfy the radiation condition at infinity. Then, to solve problem $(T)$, it is enough to show that given $A$ and $B$ in $L_{T}^{2 \text {,Div }}(\partial D)$, we can find $U$ and $V$ such that the above electric fields satisfy on $\partial D$

$$
\begin{gathered}
N \times E_{e}-N \times E_{i}=A, \\
\frac{1}{\mu_{e}} N \times \operatorname{curl} E_{e}-\frac{1}{\mu_{i}} N \times \operatorname{curl} E_{i}=B .
\end{gathered}
$$

That is, we need to solve the system

$$
\begin{aligned}
& \mu_{e}\left(-\frac{1}{2} I+M_{e}\right) U+L_{e} V-\mu_{i}\left(\frac{1}{2} I+M_{i}\right) U-L_{i} V=A \\
& L_{e} U+\frac{k_{e}^{2}}{\mu_{e}}\left(-\frac{1}{2} I+M_{e}\right) V-L_{i} U-\frac{k_{i}^{2}}{\mu_{i}}\left(\frac{1}{2} I+M_{i}\right) V=B .
\end{aligned}
$$

We rewrite this as

$$
\begin{gathered}
\left(\begin{array}{cc}
-\frac{\mu_{e}+\mu_{i}}{2} I+\mu_{e} M_{e}-\mu_{i} M_{i} & \begin{array}{c}
L_{e}-L_{i} \\
L_{e}-L_{i}
\end{array} \\
-\left(\frac{k_{e}^{2}}{2 \mu_{e}}+\frac{k_{i}^{2}}{2 \mu_{i}}\right) I+\frac{k_{e}^{2}}{\mu_{e}} M_{e}-\frac{k_{i}^{2}}{\mu_{i}} M_{i}
\end{array}\right) \cdot\left(\begin{array}{c}
U \\
V
\end{array}\right) \\
=\left(\begin{array}{c}
A \\
B
\end{array}\right) .
\end{gathered}
$$

Notice that the above system, originally defined in the the product space $L_{T}^{2 \text {,Div }}(\partial D) \times$ $L_{T}^{2 \text {,Div }}(\partial D)$, makes sense in the space $L_{T}^{2}(\partial D) \times L_{T}^{2}(\partial D)$. Now, we observe that if $M_{0}$ is the potential-theoretic version of $M$, the above matrix of operators can be decompose as the sum of two matrices, $\mathrm{W}_{1}+\mathrm{W}_{2}$, where

$$
\mathrm{W}_{1}=\left(\begin{array}{cc}
-\frac{\mu_{e}+\mu_{i}}{2} I+\left(\mu_{e}-\mu_{i}\right) M_{0} & 0 \\
0 & -\left(\frac{k_{e}^{2}}{2 \mu_{e}}+\frac{k_{i}^{2}}{2 \mu_{i}}\right) I+\left(\frac{k_{e}^{2}}{\mu_{e}}-\frac{k_{i}^{2}}{\mu_{i}}\right) M_{0}
\end{array}\right)
$$

and

$$
\mathrm{W}_{2}=\left(\begin{array}{cc}
\frac{\mu_{e}}{2}\left(M_{e}-M_{0}\right)+\frac{\mu_{i}}{2}\left(M_{0}-M_{i}\right) & L_{e}-L_{i} \\
L_{e}-L_{i} & \frac{k_{i}^{2}}{\mu_{e}}\left(M_{e}-M_{0}\right)+\frac{k_{i}^{2}}{\mu_{i}}\left(M_{0}-M_{i}\right)
\end{array}\right) .
$$

Since we are assuming that

$$
\frac{\mu_{e}+\mu_{i}}{\mu_{e}-\mu_{i}} \quad \text { and } \quad \frac{\frac{k_{e}^{2}}{\mu_{e}}+\frac{k_{i}^{2}}{\mu_{i}}}{\frac{k_{e}^{2}}{\mu_{e}}-\frac{k_{i}^{2}}{\mu_{i}}}=\frac{\epsilon_{e}+\epsilon_{i}}{\epsilon_{e}-\epsilon_{i}}
$$

are not in the interval $[-1,1]$, Lemma 4.6 implies that the matrix $\mathrm{W}_{1}$ is an invertible operator in both $L_{T}^{2}(\partial D) \times L_{T}^{2}(\partial D)$ and $L_{T}^{2, \text { Div }}(\partial D) \times L_{T}^{2 \text {,Div }}(\partial D)$. On the other hand, by Lemma 4.5 , the matrix $\mathrm{W}_{2}$ is compact in $L_{T}^{2}(\partial D) \times L_{T}^{2}(\partial D)$ and maps this space 
into $L_{T}^{2 \text {,Div }}(\partial D) \times L_{T}^{2 \text {,Div }}(\partial D)$. From this follows that the matrix $\mathrm{W}_{1}+\mathrm{W}_{2}$ has index zero in $L_{T}^{2}(\partial D) \times L_{T}^{2}(\partial D)$. It also follows that if $U$ and $V$ are solutions of the system of boundary integral equations in $L_{T}^{2}(\partial D) \times L_{T}^{2}(\partial D)$, then $U$ and $V$ are in $L_{T}^{2 \text {,Div }}(\partial D)$ if and only if $A$ and $B$ are in $L_{T}^{2, \text { Div }}(\partial D)$. In particular, the null space of the matrix $\mathrm{W}_{1}+\mathrm{W}_{2}$ is the same in both spaces. If we can show that this matrix of operators is one-to-one in $L_{T}^{2}(\partial D) \times L_{T}^{2}(\partial D)$, we will have by the previous observation that it is invertible in $L_{T}^{2}(\partial D) \times L_{T}^{2}(\partial D)$ and also in $L_{T}^{2 \text {,Div }}(\partial D) \times L_{T}^{2 \text {,Div }}(\partial D)$. This will conclude the proof of the theorem.

Assume that $U$ and $V$ are solutions of the system with $A=B=0$. Since $U$ and $V$ must be in $L_{T}^{2, \text { Div }}(\partial D), E_{i}, H_{i}$ and $E_{e}, H_{e}$ are solutions of the homogeneous version of problem $(T)$, and by Theorem 3.4, they must be identically zero. In particular, on the boundary of the domain,

$$
N \times E_{i}=N \times E_{e}=N \times \operatorname{curl} E_{i}=N \times \operatorname{curl} E_{e}=0 .
$$

Now consider the new vector fields

$$
\begin{gathered}
E_{e}^{\prime}(X)=-\operatorname{curl} \mathcal{S}_{i} U(X)-\frac{1}{\mu_{i}} \operatorname{curl} \operatorname{curl} \mathcal{S}_{i} V(X) \text { in } \mathrm{R}^{3} \backslash \bar{D}, \\
E_{i}^{\prime}(X)=\operatorname{curl} \mathcal{S}_{e} U(X)+\frac{1}{\mu_{e}} \operatorname{curl} \operatorname{curl} \mathcal{S}_{e} V(X) \text { in } D .
\end{gathered}
$$

Going to the boundary, we obtain the trace values,

$$
\begin{aligned}
& N \times E_{e}^{\prime}=\left(\frac{1}{2} I-M_{i}\right) U-\frac{1}{\mu_{i}} L_{i} V, \\
& N \times E_{i}^{\prime}=\left(\frac{1}{2} I+M_{e}\right) U+\frac{1}{\mu_{e}} L_{e} V,
\end{aligned}
$$

and

$$
\begin{gathered}
N \times \operatorname{curl} E_{e}^{\prime}=-L_{i} U-\frac{k_{i}^{2}}{\mu_{i}}\left(-\frac{1}{2} I+M_{i}\right) V, \\
N \times \operatorname{curl} E_{i}^{\prime}=L_{e} U+\frac{k_{e}^{2}}{\mu_{e}}\left(\frac{1}{2} I+M_{e}\right) V .
\end{gathered}
$$

It follows that on $\partial D$,

$$
N \times E_{e}^{\prime}-N \times E_{i}^{\prime}=-\frac{1}{\mu_{i}} N \times E_{i}-\frac{1}{\mu_{e}} N \times E_{e}=0
$$

and also

$$
\frac{\mu_{i}}{k_{i}^{2}} N \times \operatorname{curl} E_{e}^{\prime}-\frac{\mu_{e}}{k_{e}^{2}} N \times \operatorname{curl} E_{i}^{\prime}=-\frac{1}{k_{i}^{2}} N \times \operatorname{curl} E_{i}-\frac{1}{k_{e}^{2}} N \times \operatorname{curl} E_{e}=0
$$

Therefore, $E_{i}^{\prime}$ and $E_{e}^{\prime}$ are solutions of problem $\left(T^{\prime}\right)$ and, by Theorem 3.5 , they must be identically zero too. In particular,

$$
N \times E_{i}^{\prime}=N \times E_{e}^{\prime}=N \times \operatorname{curl} E_{i}^{\prime}=N \times \operatorname{curl} E_{e}^{\prime}=0
$$


using the trace results of the previous section. Finally,

$$
U=N \times E_{i}+\mu_{i} N \times E_{e}^{\prime}=0,
$$

and

$$
-V=N \times E_{e}-\mu_{e} N \times E_{i}^{\prime}=0 .
$$

In physical applications, the parameters $\mu_{i}$ and $\mu_{e}$ are usually assumed to be real numbers. In such a case, the conditions on the electromagnetic parameters take the following simpler form.

THEOREM 5.2. Let $D$ be a $C^{1}$ domain in $R^{3}$. Let $\operatorname{Im} k_{i}>0$ and $\operatorname{Im} k_{e}>0$, and assume that $\mu_{i}, \mu_{e}$, and $\omega$ are positive numbers. Then the transmission problem $(T)$ has a unique solution for any $A$ and $B$ in $L_{T}^{2, \text { Div }}(\partial D)$.

Proof. First, observe that if $\mu_{i}$ and $\mu_{e}$ are positive numbers, then

$$
\left|\frac{\mu_{e}+\mu_{i}}{\mu_{e}-\mu_{i}}\right|>1
$$

and if $\omega$ is a positive number, then the conditions $\operatorname{Im} k_{i}>0$ and $\operatorname{Im} k_{e}>0$ imply that

$$
\left|\frac{\epsilon_{e}+\epsilon_{i}}{\epsilon_{e}-\epsilon_{i}}\right|>1
$$

Also, conditions (13) and (14) are trivially satisfied. It follows that to use Theorem 3.5, we need only to check that either

$$
\operatorname{Im}\left(k_{i} \bar{k}_{e}^{2}\right) \leq 0
$$

or

$$
\operatorname{Im}\left(\frac{\epsilon_{i}}{\epsilon_{e}}\right)>0 \text { and } \operatorname{Re}\left(\frac{\epsilon_{i}}{\epsilon_{e}}\right)>0 .
$$

By writing

$$
\begin{aligned}
\epsilon_{i} & =\left|\epsilon_{i}\right| \exp \left(i \arctan \frac{\sigma_{i}}{\omega \epsilon_{0 i}}\right), \\
\epsilon_{e} & =\left|\epsilon_{e}\right| \exp \left(i \arctan \frac{\sigma_{e}}{\omega \epsilon_{0 e}}\right)
\end{aligned}
$$

we see that (16) is equivalent to

$$
\frac{\sigma_{i}}{2 \epsilon_{0 i}} \leq \frac{\sigma_{e}}{\epsilon_{0 e}}
$$

On the other hand, for positive $\omega$, the real part of $\epsilon_{i} / \epsilon_{e}$ is always positive, and a computation shows that (17) becomes equivalent to

$$
\frac{\sigma_{i}}{\epsilon_{0 i}}>\frac{\sigma_{e}}{\epsilon_{0 e}} .
$$

Obviously, either (18) or (19) is satisfied, which concludes the proof.

Finally, the proof of Theorem 5.1 shows that for a Lipschitz domain $D$ the following result holds.

THEOREM 5.3. Let $D$ be a Lipschitz domain in $R^{3}$. Assume that the electromagnetic parameters satisfy the conditions in Theorem 3.5. Assume also that $\frac{\mu_{e}+\mu_{i}}{\mu_{e}-\mu_{i}}$ and $\frac{\epsilon_{e}+\epsilon_{i}}{\epsilon_{e}-\epsilon_{i}}$ are not in the spectrum of $2 M_{0}$ as an operator in $L_{T}^{2}(\partial D)$. Then the transmission problem $(T)$ has a unique solution for any $A$ and $B$ in $L_{T}^{2, \text { Div }}(\partial D)$. 
Acknowledgment. The author would like to thank Grant Welland for many useful conversations in the subject.

\section{REFERENCES}

[1] R. BROWN AND Z. SHEN, The initial-Dirichlet problem for a fourth-order parabolic equation in Lipschitz cylinders, Indiana Univ. Math. J., 39 (1990), pp. 1313-1353.

[2] A. Calderón, Cauchy integral on Lipschitz curves and related operators, Proc. Nat. Acad. Sci. U.S.A., 74 (1977), pp. 1324-1327.

[3] R. Colfman, A. McIntosch, And Y. Meyer, L'intégrale de Cauchy définit un opérateur borné sur $L^{2}$ pour les courbes Lipschitiziennes, Ann. of Math., 116 (1982), pp. 361-387.

[4] D. Colton And R. Kress, Integral Equation Methods in Scattering Theory, John Wiley, New York, 1983.

[5] M. Costabel And E. Stephan, Strongly elliptic boundary integral equations for electromagnetic transmission problems, Proc. Roy. Soc. Edinburgh Sect. A, 109 (1988), pp. 271-296.

[6] — Integral equations for transmission problems in linear elasticity, J. Integral Equations Appl., 2 (1990), pp. 211-223.

[7] B. Dahlberg, C. Kenig, and G. Verchota, Boundary value problems for the systems of elastostatics in Lipschitz domains, Duke Math. J., 57 (1988), pp. 795-818.

[8] L. EscauriazA, E. FABES, AND G. Verchota, On a regularity theorem for weak solutions to transmission problems with internal Lipschitz boundaries, Proc. Amer. Math. Soc., 115 (1992), pp. 1069-1076.

[9] E. FABes, M. Jodeit, AND N. Rivière, Potential techniques for boundary value problems on $C^{1}$ domains, Acta Math., 141 (1978), pp. 165-186.

[10] E. FABES, M. SAND, AND J. SEO, The spectral radius of the classical layer potentials on convex domains, in Partial Differential Equations with Minimal Smoothness and Applications, Springer-Verlag, New York, 1992, pp. 129-137.

[11] D. Jerison And C. Kenig, The Neumann problem on Lipschitz domains, Bull. Amer. Math. Soc., 4 (1981), pp. 203-207.

[12] W. KupRaDSE, Randewertaufgaben der Schwngungstheorie und Integralgleichungen, Deutscher Verlag der Wissenschafen, Berlin, 1956.

[13] M. Mitrea, R. Torres, AND G. Welland, Regularity and approximation results for the Maxwell problem in $C^{1}$ and Lipschitz domains, in Clifford Algebras in Analysis and Related Topics, CRC Press, Boca Raton, 1995, pp. 297-308.

[14] - Layer potential techniques in electromagnetism, preprint.

[15] C. Müller, Foundations of the Mathematical Theory of Electromagnetic Waves, SpringerVerlag, Berlin, 1969.

[16] R. TORRES AND G. Welland, The Helmholtz equation and transmission problems with Lipschitz interfaces, Indiana Univ. Math. J., 42 (1993), pp. 1457-1485.

[17] G. Verchota, Layer potentials and boundary value problems for Laplace's equation in Lipschitz domains, J. Funct. Anal., 59 (1984), pp. 572-611.

[18] - The Dirichlet problem for the biharmonic equation in $C^{1}$ domains, Indiana Univ. Math. J., 36 (1987), pp. 867-895.

[19] T. von Petersdorff, Boundary integral equations for mixed Dirichlet, Neumann and transmission problems, Math. Methods Appl. Sci., 11 (1989), pp. 185-213.

[20] P. WILDE, Transmission problems for the vector Helmholtz equation, Proc. Roy. Soc. Edinburgh Sect. A, 105 (1987), pp. 61-76. 